\title{
Apple chlorotic fruit spot viroid: a putative new pathogenic viroid on apple characterized by next-generation sequencing
}

\author{
Thomas Leichtfried $^{1} \cdot$ Stefanie Dobrovolny ${ }^{3} \cdot$ Helga Reisenzein $^{1} \cdot$ Siegrid Steinkellner $^{2}$ (i) $\cdot$ Richard A. Gottsberger $^{1}$
}

Received: 20 June 2019 / Accepted: 29 August 2019 / Published online: 9 October 2019

(c) The Author(s) 2019

\begin{abstract}
Viroid-like symptoms were observed in 2016 on apple fruits of the cultivar "Ilzer Rose" in southern Burgenland, Austria. Preliminary molecular biological investigations indicated that the symptoms were caused by a new unknown viroid. Therefore, new primers were designed, and the whole genome sequence of the viroid (354 nt) was determined by next-generation amplicon sequencing using the Illumina MiSeq ${ }^{\circledR}$ platform (San Diego, California, USA). The viroid secondary structure has a rod-like conformation and contains conserved regions (the TCR, CCR upper strand, and CCR lower strand) that are characteristic of members of the genus Apscaviroid. Based on our results and the demarcation criteria for viroids, the tentatively named "apple chlorotic fruit spot viroid" should be considered a putative new member of the genus Apscaviroid.
\end{abstract}

\section{Introduction}

Apple is an important fruit with a high economic impact and is also the most important fruit crop in Austria [1]. Viroids can cause evident fruit symptoms on pome fruits and can cause economic losses by reducing fruit yield and quality, thus resulting in unmarketable fruits [2]. More than 30 plantpathogenic viroids are known thus far. In apple, three plantpathogenic viroids, all belonging to the genus Apscaviroid, have been described: apple scar skin viroid (ASSVd), apple dimple fruit viroid (ADFVd), and the unassigned apple fruit crinkle viroid (AFCVd) [3]. Recently, a new technique, nextgeneration sequencing (NGS), was developed, and this can be used as an important tool for the discovery [4-7], detection, identification, and characterization [8] of new plant

Handling Editor: F. Murilo Zerbini.

Electronic supplementary material The online version of this article (https://doi.org/10.1007/s00705-019-04420-9) contains supplementary material, which is available to authorized users.

Siegrid Steinkellner

siegrid.steinkellner@boku.ac.at

1 Institute for Sustainable Plant Protection, Austrian Agency for Health and Food Safety, 1220 Vienna, Austria

2 Institute of Plant Protection, University of Natural Resources and Life Sciences Vienna, 3430 Tulln an der Donau, Austria

3 Institute for Food Safety, Austrian Agency for Health and Food Safety, 1220 Vienna, Austria viroids and viruses $[9,10]$. The major advantage of NGS is the ability to generate an immense amount of data in a short time; thus, it is a cost-effective method to obtain extensive genome information rapidly. In 2016, viroid-like symptoms were observed on apple fruits of the local cultivar "Ilzer Rose" in the Austrian province Burgenland (Fig. 1A). In this paper, we report the detection and identification of the whole genome of this putative new pathogenic viroid on apple using NGS amplicon sequencing.

\section{Materials and methods}

For the first screening, total RNA was extracted directly from symptomatic apple skins (cv. "Ilzer Rose") using an RNeasy Plant Mini Kit (QIAGEN, Hilden, Germany), and the generic primers PBCV100C and PBCV194H were used for the detection of pome fruit viroids [11]. Based on the generic sequences, two primer sets were designed to sequence the whole genome and to confirm the circular form of the unknown viroid by NGS (primer set 1, ACFSVd_F1 [5'-CTGAGATTGGCTCGAGGAGTCC-3'] and ACFSVd_R1 [5'-GCGAGTTCTGGACACGAGAG-3']; primer set 2, ACFSVd_F2 [5'-CCGCCTTTTTCTCTATCC TC-3'] and ACFSVd_R2 [5'-AGCAGGCGAGAACTGGAC AC-3']) (Supplementary Fig. S1).

DNA library preparation was performed in a two-step PCR process with a dual-indexing principle according the "16S Metagenomics Sequencing Library Preparation" 


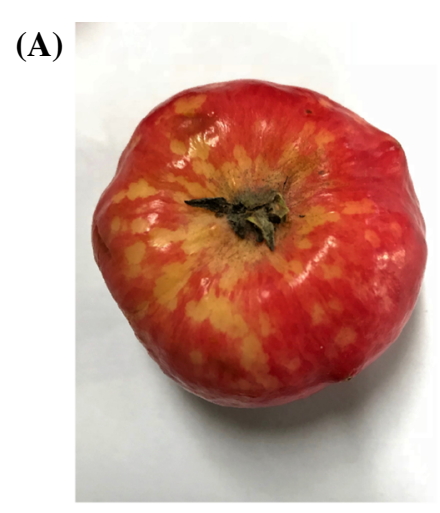

(B)

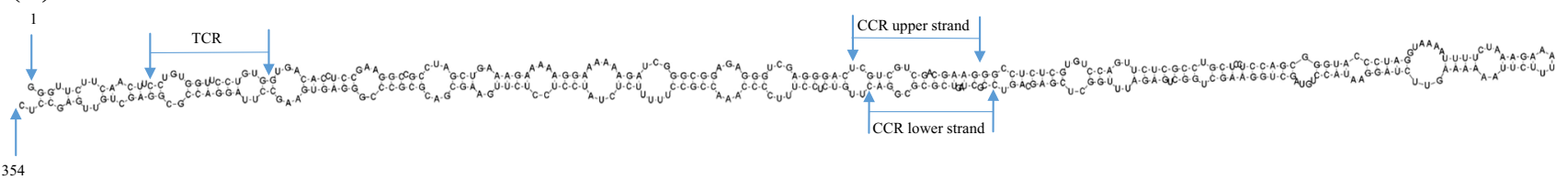

Fig. 1 (A) Yellow and chlorotic fruit spots and peak-like symptoms on the skin of apples of cv. "Ilzer Rose" caused by ACFSVd. (B) Nucleotide sequence and proposed secondary structure of ACFSVd. Structures are represented for the lowest-energy form at $37{ }^{\circ} \mathrm{C}$ pre-

protocol from Illumina and modifications described by Dobrovolny et al. [12] The sequencing reaction was performed on an Illumina MiSeq ${ }^{\circledR}$ platform (San Diego, California, USA) using a 300-cycle MiSeq ${ }^{\circledR}$ Reagent Kit. Further data analysis and processing steps were implemented in the Galaxy workflow $[12,13]$. The de novo assembly of the obtained sequences was performed using Geneious ${ }^{\circledR}$ software (Version 10.1.3).

Phylogenetic analysis was carried out based on whole genome sequences (range, 269-401 nt) of members of the genus Apscaviroid. The reference sequences, except ACF$\mathrm{SVd}$, were retrieved from NCBI GenBank. A multiple alignment was generated using the Geneious algorithm (similar to the Clustal W algorithm) in Geneious ${ }^{\circledR}$ software (Version 10.1.3)

\section{Results and discussion}

After the reads were processed via a quality check and the Galaxy workflow, the number of sequences obtained from the Illumina MiSeq ${ }^{\circledR}$ platform with primer set 1 was 145,679 reads, while the number of sequences obtained with primer set 2 was 21,160 reads. The operational taxonomic unit (OTU) with the largest number of identical sequences was used to assemble the genome sequence of ACFSVd using Geneious ${ }^{\circledR}$ software (Version 10.1.3). The whole-genome data have been deposited in the databases provided by NCBI dicted using the model of Turner and Mathews [18], created using Geneious ${ }^{\circledR}$ software (Version 10.1.3). The first and the last nucleotide of ACFSVd, the TCR and the CCR lower and upper strands are indicated by arrows

(accession no. MF521431.2). The whole genome of ACF$\mathrm{SVd}$ is $354 \mathrm{nt}$ long (Fig. 1B). The circular RNA molecule consists of 76 A (21.5\%), $95 \mathrm{C}(26.8 \%), 97 \mathrm{G}(24.3 \%)$ and $86 \mathrm{U}(24.3 \%)$ with a G+C content of $54.2 \%$ and a free energy of $-147.40 \mathrm{kcal} / \mathrm{mol}$ (Fig. 1B). The characteristic features of the secondary structure of the genome of members of the genus Apscaviroid are the terminal conserved regions (TCRs), the upper and lower central conserved regions (CCRs), and a quasi-rod-like conformation (Fig. 1B) [14]. No mismatches were observed between the TCR region and CCR upper strand of ACFSVd and those of the apscaviroid ASSVd type reference (NC_001340). There was only one mismatch on the upper strand (Table 1). The sequence was compared to sequences in the NCBI GenBank database by BLASTn analysis. This RNA molecule is most similar to grapevine speckle viroid $2(80.8 \%$ ) (accession no. FJ597935.1) and grapevine speckle viroid 1 (76.7 \%) (accession no. KF007313.1), but the similarity is limited to a short fragment. Phylogenetic analysis supported the BLAST results. ACFSVd formed a monophyletic group with grapevine speckle viroid 1 and grapevine speckle viroid 2, and the genetic distance revealed that ACFSVd is clearly a new member of the genus Apscaviroid (Fig. 2). ACFSVd differs from other confirmed apple viroids in the genus Apscaviroid, such as ASSVd and ADFVd, in the symptoms it causes in apple fruits. The symptoms of ACFSVd infection are yellow chlorotic spots and pronounced peak-like deformations on apple fruits (Fig. 1A). In contrast, ASSVd causes scarred 
Table 1 Terminal conserved region (TCR) and central conserved region (CCR) sequences of ACFSVd and ASSVd, the type member of the genus Apscaviroid. One mismatch (underlined and bold) was observed in the CCR lower strand. GenBank accession numbers are shown in brackets

\begin{tabular}{llll}
\hline & TCR & CCR upper strand & CCR lower strand \\
\hline ASSVd (NC_001340) & CNNGNGGUUCCUGUGG & UCGUCGUCGACGAAGG & CCGCUAGUCGAGCGGAC \\
ACFSVd (MF521431.2) & CCUGUGGUUCCUGUGG & UCGUCGUCGACGAAGG & CCGCUAGUCGCGCGGAC \\
\hline
\end{tabular}

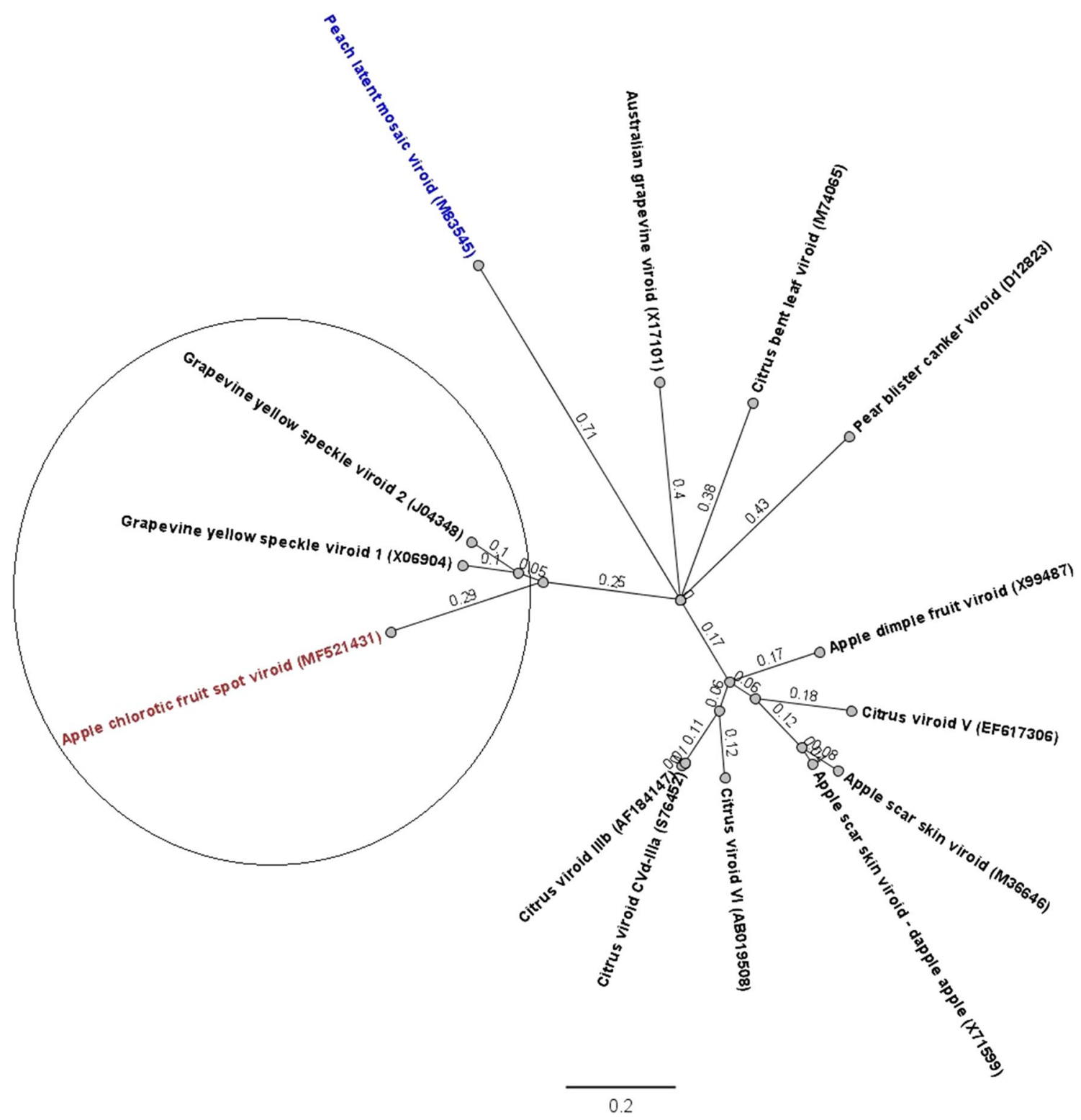

Fig. 2 Phylogenetic analysis of ACFSVd including all assigned members of the genus Apscaviroid and peach latent mosaic viroid as an outgroup (labelled in blue). The multiple sequence alignment was generated using Geneious ${ }^{\circledR}$ software (version 10.1.3). The phylogenetic tree was constructed by the neighbour-joining $(\mathrm{NJ})$ clustering

skin or speckle symptoms on apple fruits [15], and ADFVd induces yellow-green spots, which are sometimes slightly method [19], and 5,000 bootstrap replicates were performed. The genetic distance was calculated using the Tamura-Nei model [20]. ACFSVd is indicated in red and bold. The scale represents 0.2 nucleotide substitutions per site. The circle indicates the cluster of adjacent relatives. GenBank accession numbers are shown in brackets

depressed (dimpled) and scattered mainly around the calyx [16]. To test the transmissibility of the RNA molecule, 
scions were grafted from the symptomatic apple tree cultivar Ilzer Rose to three apple trees of the cultivar Gala. These trees were tested positive distant from the graft after half a year using RT-PCR with the ACFSVd-specific primers ACFSVd-F (5'-CTAGTCGCGCGGACTTGTCTC-3') and ACFSVd-R (5'-CGAGAACTGGACACGAGAGG-3').

Analyses of the complete nucleotide sequence indicated that ACFSVd is a putative new viroid in the genus Apscaviroid according to the demarcation criteria from the International Committee on Taxonomy of Viruses (ICTV) [14, 17]. The whole genome sequence was used to generate a phylogenetic tree. The evaluation of the data showed that the "closest" relatives are grapevine speckle viroid 1 and 2. ACFSVd is transmissible by grafting. Further pathways are still under investigation.

In summary, in this study, a putative new unknown pathogenic viroid of apple was discovered and identified using NGS technology. The species demarcation criteria for viroids indicate that ACFSVd should be regarded as a new distinct member of the genus Apscaviroid.

Acknowledgements Open access funding provided by University of Natural Resources and Life Sciences Vienna (BOKU). The authors gratefully thank Johann Schaffer and Norbert Berger (Austrian Agency for Health and Food Safety, Institute for Sustainable Plant Protection, Vienna) for helping with RNA extraction and the sampling procedure.

\section{Compliance with ethical standards}

Conflict of interest The authors declare that they have no conflict of interest.

Ethical approval This research did not contain studies involving human participants or animals by any of the authors.

Open Access This article is distributed under the terms of the Creative Commons Attribution 4.0 International License (http://creativeco mmons.org/licenses/by/4.0/), which permits unrestricted use, distribution, and reproduction in any medium, provided you give appropriate credit to the original author(s) and the source, provide a link to the Creative Commons license, and indicate if changes were made.

\section{References}

1. Statistik Austria: Obstproduktion aus Erwerbsanlagen (2018). https://www.statistik.at/web_de/statistiken/wirtschaft/land_und_ forstwirtschaft/agrarstruktur_flaechen_ertraege/obst/index.html. Accessed 15 May 2019

2. Hadidi A, Barba M (2011) Economic impact of pome and stone fruit viruses and viroids. In: Hadidi A, Barba M, Candresse T, Jelkmann W (eds) Virus and virus-like diseases of pome and stone fruits. The American Phytopathological Society, Minnesota, pp 1-7

3. International Committee on Taxonomy of Viruses ICTV. In: International Committee on Taxonomy of Viruses ICTV. https://talk. ictvonline.org/taxonomy/. Accessed 15 May 2019

4. Zhang Z, Qi S, Tang N et al (2014) Discovery of replicating circular RNAs by RNA-seq and computational algorithms. PLOS Pathog 10:e1004553. https://doi.org/10.1371/journal.ppat.1004553
5. Ito T, Suzaki K, Nakano M, Sato A (2013) Characterization of a new apscaviroid from American persimmon. Arch Virol 158:2629-2631. https://doi.org/10.1007/s00705-013-1772-x

6. Jakse J, Radisek S, Pokorn T et al (2015) Deep-sequencing revealed Citrus bark cracking viroid (CBCVd) as a highly aggressive pathogen on hop. Plant Pathol 64:831-842. https:// doi.org/10.1111/ppa.12325

7. Chiumenti M, Torchetti EM, Di Serio F, Minafra A (2014) Identification and characterization of a viroid resembling apple dimple fruit viroid in fig (Ficus carica L.) by next generation sequencing of small RNAs. Virus Res 188:54-59. https://doi.org/10.1016/j. virusres.2014.03.026

8. Barba M, Hadidi A (2017) Application of next-generation sequencing technologies to viroids. Viroids and satellites. Elsevier/Academic Press, London, pp 401-412

9. Shen P, Tian X, Zhang S et al (2018) Molecular characterization of a novel luteovirus infecting apple by next-generation sequencing. Arch Virol 163:761-765. https://doi.org/10.1007/s0070 5-017-3633-5

10. Wu L-P, Yang T, Liu H-W et al (2018) Molecular characterization of a novel rhabdovirus infecting blackcurrant identified by highthroughput sequencing. Arch Virol 163:1363-1366. https://doi. org/10.1007/s00705-018-3709-x

11. Sano T, Yoshida H, Goshono M et al (2004) Characterization of a new viroid strain from hops: evidence for viroid speciation by isolation in different host species. J Gen Plant Pathol 70:181-187. https://doi.org/10.1007/s10327-004-0105-Z

12. Dobrovolny S, Blaschitz M, Weinmaier T et al (2019) Development of a DNA metabarcoding method for the identification of fifteen mammalian and six poultry species in food. Food Chem 272:354-361. https://doi.org/10.1016/j.foodchem.2018.08.032

13. Afgan E, Baker D, van den Beek M et al (2016) The Galaxy platform for accessible, reproducible and collaborative biomedical analyses: 2016 update. Nucleic Acids Res 44:W3-W10. https:// doi.org/10.1093/nar/gkw343

14. Di Serio F, Flores R, Verhoeven JThJ et al (2014) Current status of viroid taxonomy. Arch Virol 159:3467-3478. https://doi. org/10.1007/s00705-014-2200-6

15. Hadidi A, Barba M, Hong N, Hallan V (2017) Apple scar skin viroid. In: Hadidi A, Flores R, Randles JW, Palukaitis P (eds) Viroids and satellites. Elsevier/Academic Press, London, pp 217-228

16. Di Serio F, Torchetti EM, Flores R, Sano T (2017) Other apscaviroids infecting pome fruit trees. In: Hadidi A, Flores R, Randles JW, Palukaitis P (eds) Viroids and satellites. Elsevier/Academic Press, London, pp 229-242

17. Flores R, Randles J, Bar-Joseph M, Diener T (1998) A proposed scheme for viroid classification and nomenclature. Arch Virol 143:623-629. https://doi.org/10.1007/s007050050318

18. Turner DH, Mathews DH (2010) NNDB: the nearest neighbor parameter database for predicting stability of nucleic acid secondary structure. Nucleic Acids Res 38:D280-D282. https://doi. org/10.1093/nar/gkp892

19. Saitou N, Nei M (1987) The neighbor-joining method: a new method for reconstructing phylogenetic trees. Mol Biol Evol 4:406-425. https://doi.org/10.1093/oxfordjournals.molbev.a0404 54

20. Tamura K, Nei M (1993) Estimation of the number of nucleotide substitutions in the control region of mitochondrial DNA in humans and chimpanzees. Mol Biol Evol 10:512-526. https://doi. org/10.1093/oxfordjournals.molbev.a040023

Publisher's Note Springer Nature remains neutral with regard to jurisdictional claims in published maps and institutional affiliations. 